\title{
Genetics of schizophrenia: communicating scientific findings in the clinical setting
}

\section{JL Roos}

Department of Psychiatry, University of Pretoria, Pretoria, South Africa

\begin{abstract}
The expected identification of susceptibility genes for psychiatric disorders may bring new opportunities and expectations from patients and families for the clinical translation of research findings in psychiatric genetics. In this article information is provided about familial risk of schizophrenia with the theory behind individualizing risk of recurrence highlighted. Recent new findings regarding the new genetic frontier, Copy Number Variations (CNV), are summarized and the genetic architecture of familial and sporadic schizophrenia applicable to the clinical situation is reviewed. A scenario in which genetic testing could be applied in velocardiofacial syndrome (VCFS) type schizophrenia is debated. Referring to genetic discrimination in mental disorders, reference is made to the implementation of the Federal Genetic Information non-discrimination Act (GINA) of 2008 in the USA and the Mental Health Care Act of 2002 in SA.
\end{abstract}

Keywords: Schizophrenia; Copy number variations; Familial risk; Individual risk; Velocardiofacial syndrome

Received: $20-05-2010$

Accepted: 25-06-2010

doi: 10.4314/ajpsy.v14i2.1

\section{Introduction}

In the coming years, the expected identification of susceptibility genes for psychiatric disorders may bring new opportunities and expectations from patients and families for the clinical translation of research findings in psychiatric genetics. There is evidence for possible increasing demand for genetic counselling, particularly if specific genes related to psychiatric disorders are identified.

One area in which familial / genetic research has already been clinically relevant is in providing information about familial risks of illness. The relative inattention to genetic counselling for psychiatric disorders is undoubtly due, in part, to the limitation of available information about genetic risks for common disorders and the inability to individualize risks (due to the absence of genetic testing). Nevertheless, perhaps related to the recent "high profile" of genetics, clinicians are increasingly confronted with questions from patients and families, regarding risks of disorders for

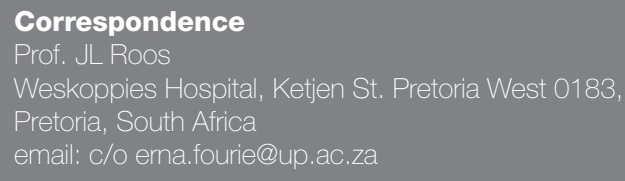

themselves or their children. The coming years will likely see significant advances in the clinical application of this knowledge in psychiatric practice. ${ }^{1}$

As scientific advances allow more accurate identification of individual genetic risks, increasing numbers of people will be seeking this kind of help. But professional with genetic expertise often have a poor understanding of psychiatric diagnoses. Given that genetic counselling for complex disorders is among the most challenging topics encountered by genetics professionals, gaps in genetic knowledge may become a major limiting factor in psychiatrists' ability to provide counselling. Hence, better professional education will be needed to improve patient and family education about the genetics of psychiatric disorders. ${ }^{1}$

Having a family history of the disorder is currently the best predictor for the development of several disorders, including schizophrenia and bipolar disorder. Does our current state of knowledge regarding the genetics of schizophrenia support the clinical application of this knowledge in the form of genetic counselling?

Being involved with the genetic study of schizophrenia in the Afrikaner for more than 10 years, I have been approached by family members and patients alike for diagnostic, preventive and reproduction purposes for genetic testing and counselling for schizophrenia. This paper will address 
aspects of the current status of knowledge regarding the genetics of schizophrenia and some of the issues involved in conveying genetic information in the clinical setting.

\section{Intersection of Genetics and Clinical Psychiatry}

For most psychiatrists, genetic counselling and the role of genetic contributions to psychiatric illness are unlikely to be a frequent clinical concern. However the internet provides fast, easy and sometimes unreliable medical information e.g. on the genetics of schizophrenia. Patients might engage their psychiatrists in this regard. An awareness of genetic contributions to psychiatric symptoms can at times, have important implications for diagnosis and patient care. For example, the diagnosis and treatment of a patient with firstepisode psychosis could be crucially affected by the determination that she has a family history of acute intermittent porphyria. This genetic disorder can present with psychosis and be one where certain psychotropic medications may precipitate acute symptoms. Less dramatically, a strong family history of bipolar disorder might influence drug selection for a patient who present with a first episode psychosis. The two scenarios also highlight a crucial distinction between mendelian, single-gene disorders and complex (polygenic and multifactorial) disorders. Single-gene disorders (such as Huntington's disease) typically follow classical Mendelian patterns of inheritance (e.g. recessive, dominant) and are often highly penetrant (that is, the risk of illness in those carrying the genetic liability is high). In contrast, most cases of psychiatric illness are believed to be genetically "complex". For these disorders, the onset of illness may require the additive or interactive effect of multiple genes; each individual gene contributing a small amount to risk. In addition, additive or interactive effects of environmental risk factors (e.g. stressful life events, smoking of marijuana) may also contribute to the risk. For most types of psychiatric illness, genetic variants may represent risk factors for onset of disease, conferring susceptibility to a disorder without being sufficient to cause it. In addition to making the search for susceptibility genes very difficult, the small individual effects of these genes means that, even if we can identify the genes, the feasibility and value of genetic testing may be limited. ${ }^{1}$

\section{What is the potential role of genetic counselling in psychiatry?}

Genetic counselling for psychiatric disorders would be primarily concerned with informing individuals about the role of genetic factors in psychiatric illness, estimating recurrence risks based on family history data, and counselling based on these considerations. ${ }^{1}$

Genetic counselling offers an opportunity to reduce damaging misattributions of etiology. Such interventions must be handled with care to avoid engendering other ideas that may be equally misguided and distressing - for example a sense of guilt at having "transmitted" an illness, or fatalistic ideas about genetic determinism. ${ }^{2}$ Genetic counselling may also clarify misperceptions of recurrence risk for affected individuals and their family. If they have overestimated the risk they may be reassured. If they have underestimated the risk, they will suitably informed. ${ }^{1}$

Counselling may have other benefits, including the opportunity to talk about the experience of having a psychiatrically ill family member. The counselling session may be the first opportunity, for unaffected family members, to meet with a medical professional to discuss the disorder of interest. Affected individuals may also wish to discuss the implications of familial psychiatric illness. This education may alter perceptions of disease by dispelling inaccurate beliefs about the course, prognosis and treatment options available for mental illness. The potential risks associated with conveying risk information also need to be considered, such as discrimination (by health or life insurance companies or by employers) increased stigmatization and raising anxiety regarding test results that indicate elevated risk for disease in oneself or family members. ${ }^{3}$

\section{Providing recurrence risk for schizophrenia}

Emperic risk estimates are used for multifactorial disorders, in which the inheritance patterns are unknown and do not appear to follow the typical mendelion pedigree patterns. Currently, the major genes contributing to mental illness remain to be conclusively established and genetic testing is not yet available. For this reason, recurrence risk estimates are based on empiric risks. (typically, risks based on data from available family studies). ${ }^{1}$

By their very nature, empiric risks are summary estimates and thus would not be expected to apply directly to individual families. These risks are based on particular ascertained study populations and are not specific to the family in question. In addition, the use of our current diagnostic system may result in the inclusion of a heterogeneous group of disorders that are combined under shared diagnostic labels. This again makes it unlikely that recurrence risks would apply directly to particular families. The complexity of risk estimation is compounded by the common presence of comorbidity in probands or family members since empiric risks accounting for comorbidity may not be available.

An important component of discussing risks involves education about the distinction between classic single-gene inheritance, and complex, multi-factorial inheritance. Psychiatric illness is likely due to the effect of multiple genes in concert with environmental risk factors i.e. genes may confer risk for a disorder, but the expression of the phenotype and the probability of the disorder occurring may be difficult to predict. ${ }^{1}$

When providing recurrence risk for schizophrenia one should provide a range of risks, gathered from multiple sources. Comparisons with general population risks should be offered (Table I) ${ }^{4,5,6}$ Empiric risks are usually available for

\begin{tabular}{|c|c|c|}
\hline Disorder & $\begin{array}{l}\text { General population } \\
\text { lifetime risks }\end{array}$ & $\begin{array}{l}\text { Estimated empiric FDR } \\
\text { recurrence risks }\end{array}$ \\
\hline $\begin{array}{l}\text { Schizophrenia } \\
\text { Bipolar disorder }\end{array}$ & $\begin{array}{l}\sim 1 \% \\
\sim 1-5 \%\end{array}$ & $\begin{array}{l}5 \sim 16 \% \\
4 \sim 18 \% \\
9 \sim 25 \%\end{array}$ \\
\hline
\end{tabular}




\section{Table II: Recurrence Risks for Schizophrenia based on Relationship to Affected Individual}

[Empirical risks of developing schizophrenia for relatives of a person with schizophrenia

General population

$1 \%$

First-degree Relatives

\begin{tabular}{l|l} 
Two affected parents & $\sim 45 \%$ \\
MZ twins & $40-48 \%$ \\
DZ twins & $10-17 \%$ \\
Children & $\sim 13 \%$ \\
Parents & $6-13 \%$ \\
Siblings & $6-9 \%$ \\
Half-siblings & $3-6 \%$
\end{tabular}

Second-degree Relatives

\begin{tabular}{l|l}
\hline Grandchildren & $3-5 \%$ \\
Aunts / Uncles & $2-3 \%$ \\
Nephews / nieces & $2-4 \%$ \\
First cousin & $2-3 \%$
\end{tabular}

Source: Reference ${ }^{7,8}$

simple family relationships as illustrated in Table II. ${ }^{7,8}$ Empiric risks are likely most helpful in families with relatively few affected family members and without comorbid psychiatric illnesses. As an alternative to empiric risks, theoretical recurrence risks can be derived using estimates of certain epidemiologic parameters. These theoretical risks can serve as a guide but do not represent individualized risk estimates. Another important factor in providing recurrence risks for psychiatric disorders is the issue of the accuracy of diagnoses derived from family history and the likelihood that psychiatric symptoms exist on a continuum.

\section{Recent new findings in schizophrenia genetic research}

Genetic research in schizophrenia has introduced copy number variation to the field. The content which follows includes clinically applicable findings with reference to a paper on the genetic architecture of familial schizophrenia within a subset of Afrikaner schizophrenia patients. These findings may help to elucidate familial and sporadic cases of schizophrenia during genetic counselling.

\section{Copy Number Variation (CNV), the new genetic frontier} Rare structural changes in the genome have been identified, known as copy number variations. These changes even occur in healthy people. It has been possible to identify these rare structural changes because improved technology has been developed. CNV's occur when genetic material is lost, inserted in the wrong place, or duplicated inappropriately. Most of these variations are not inherited; instead they result from spontaneous mutations in DNA, which happens by chance at conception or during development. ${ }^{9}$
Traditional cytogenetics using light microscopy, and going back over 40 years ago, first showed that variations in chromosome copy number and other structural changes could cause disease in humans. The earliest psychiatric disorder implicated was Downs syndrome caused by the presence of an additional copy of chromosome 21. Many other visible deletions and duplications were later identified associated with disease. There are also a good number of apparently benign variations present in normal individuals. The classic examples in schizophrenia are VCFS, due to a deletion at 22ql 1 associated with psychosis in $30 \%$ of cases. In a large Scottish pedigree a disruption of the DISCl gene in a balanced lq43:1 lql 4 translocation associated with schizophrenia and other forms of major mental illness were identified. ${ }^{9}$

An emerging hypothesis is that CNVs may contribute in a more significant way than previously realized to schizophrenia and other psychiatric disorders. CNVs are often unique to an individual. The fact that these structural changes often vary greatly from one person to the next may help explain why it has been so difficult to find genetic patterns for psychiatric disease. ${ }^{9}$

\section{CNV and Schizophrenia}

In recent times a series of major articles have reported associations with schizophrenia of CNV at lq21, 15ql 1.2, $15 \mathrm{ql} 3.3,16 \mathrm{pl} 1.2,22 \mathrm{ql} 2$ and neurexin 1 loci. ${ }^{10}$ What are the implications of these new findings? All the studies have strengths and weaknesses, but when combined - and in contrast to the often contradictory findings in schizophrenia DNA linkage and SNP allelic association studies - they are internally consistent and consistent with each other:

- These studies show beyond doubt that carriers of some de novo and inherited CNVs, especially when they can be shown to disrupt gene function, are at high risk of developing schizophrenia. In the great majority of cases, the schizophrenia is phenotypically indistinguishable from schizophrenia as it presents to psychiatric clinics the world over. In a small proportion there may be associated features such as learning disability or VCFS, and in these cases the schizophrenia can be considered syndromic. ${ }^{10}$

- Deletions and/or duplications of CNVs at lq21, 15q11.2, 15q13.3, 16pl 1.2 and neurexinl loci substantially increase the risk of a broad range of major psychiatric disorders in addition to schizophrenia i.e. mental retardation, autism, bipolar disorder and attention deficit/hyperactivity disorder.

- The findings help to explain why some cases of schizophrenia appear familial and other cases appear sporadic. Sporadic cases are much more likely to be caused by de novo events. The high rate of de novo events probably also explains the well-known observation of higher risk of schizophrenia in the children than in the siblings of schizophrenia proband.s. ${ }^{10}$

- These new discoveries have important implications for genetic counselling. Risk of schizophrenia in siblings of individuals with schizophrenia is far less if the proband carries de novo CNV mutation. Many CNVs disrupt several genes, only one of which may predispose to 
schizophrenia. Others may however impact on the physical health of individuals with schizophrenia and could in part explain the poorer health outcomes associated with schizophrenia. ${ }^{10}$

- CNVs account for $2-4 \%$ of schizophrenia's overall genetic burden. As many more novel loci are identified by newer high-resolution platforms, they may account for $10-20 \% \cdot{ }^{10}$

- Determining the precise nature of the CNVs and what the genetic/epigenetic and environmental factors are that influence their penetrance and expressivity and cause them to increase risk for a broad range of neurodevelopmental disorders will be an exciting challenge for neuropsychiatric research in coming years. ${ }^{10}$

\section{Genetic architecture of Familial schizophrenia}

The strongest predictor of schizophrenia is having an affected first degree relative. In addition to the familial forms, nonfamilial (sporadic) forms of the disease also exist. The exact proportion of each form is largely unknown, but it is thought that at least $60 \%$ of cases are sporadic. ${ }^{11}$

Genome-wide linkage scans have been conducted to identify loci harbouring relatively rare mutations / variants that increase susceptibility to familial schizophrenia. Loci have been identified on almost every chromosome, but only a few regions have been replicated across studies. One such region is near the telomere of chromosome 13q This region has also been linked to bipolar disorder. In addition to linkage studies, a number of earlier as well as more recent studies have provided strong evidence supporting the importance of rare structural mutations / variants in schizophrenia vulnerability. Rare inherited structural lesions are expected to be prominent in familial schizophrenia. The full contribution of these structural lesions to transmitted liability in familial schizophrenia cases was not examined in a systematic manner until the study by Xu et al undertaken amongst the Afrikaner population in South Africa (SA). ${ }^{11}$

In addition to the genetic homogeneity, the Afrikaners are valuable for genetic studies because they present a close-knit family structure and offer the potential to perform detailed genealogical analysis. This aspect affords reliable discrimination of familial and nonfamilial forms of the disease and facilitates family-based genetic studies.

The results highlight the difference in the genetic architecture of the familial and sporadic forms of the disease. ${ }^{11}$ [See Fig 1] Based on the results of this study and previous ones, there is considerable evidence for linkage to $13 \mathrm{q}$. The findings support the notion that multiple genetic variants, including individually rare ones (often unique to a single patient), that affect many different genes contribute to the genetic risk of familial schizophrenia. This heterogeneity (present to some degree even in founder populations) is consistent with the hypothesis that there are many genes that contribute to schizophrenia and may account for past and present difficulties in finding bona fide genetic variants. These results are likely to have general implications regarding the genetic architecture of schizophrenia as there are significant clinical similarities of schizophrenia cases diagnosed in the Afrikaners and those diagnosed in more heterogeneous populations (such as in the United States of America- USA). ${ }^{11}$

Those with schizophrenia from families with no history of the illness were found to harbour eight times more spontaneous mutations than in healthy controls. These mutations were mostly in pathways affecting brain development. ${ }^{12}$ The findings strongly suggest that rare, spontaneous mutations likely contribute to vulnerability in case of schizophrenia from previously unaffected families. This may also shed light on why the illness has frustrated efforts to implicate gene variants with major effects, and seems to defy natural selection by persisting in the population even though relatively few of those affected have children. There now exists a dramatic demonstration that genetic vulnerabilities for these illnesses may not be inherited from parents, at least in the sense that these vulnerabilities were not present in the parenteral genome. ${ }^{12}$

Although it is known that genetics play a major role in the transmission of both autism and schizophrenia, most cases are sporadic rather than familial. ${ }^{12}$

\section{Figure 1: Genetic architecture of schizophrenia}

\section{Familial cases}

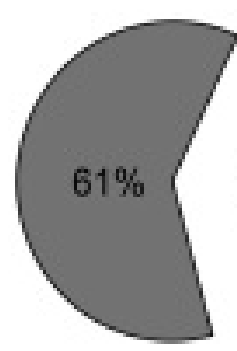

$\square$ no rare CNV

rare inhereted CNV

\section{Sporadic cases}

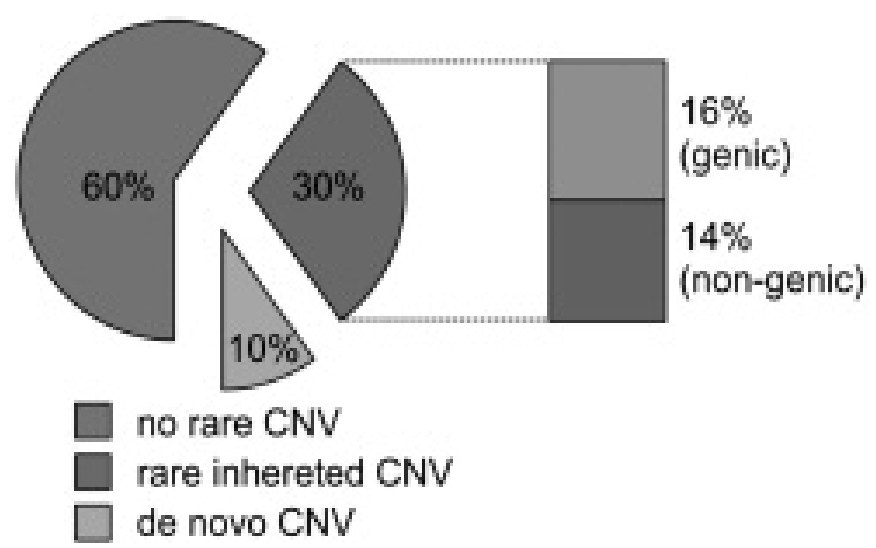




\section{Individualizing recurrence risks}

Recurrence risk assessment is more challenging to provide in the context of complex psychiatric disorders. In these cases, empiric recurrence risk (of varying quality and comprehensiveness) are often available and can be used as a basis for individualizing recurrence risks.

\section{Limitations of empiric risks for psychiatric disorders: the theory behind individualized assessment}

Empiric recurrence risk figures are the foundations on which individualized risk assessment can be developed however they do have a number of limitations. There are a limited number of family relationships for which empiric risks are available. There are limited data when there are multiple disorders within a family, or when relatives on both maternal and paternal sides are affected. Because risks for psychiatric disorders vary according to a number of factors that are typically not taken into account during the generation of empiric risks, they should generally not be applied without critical assessment. ${ }^{13}$

As with any complex disorder individualized recurrence risks should be produced by adjustment of empiric data based on characteristics including: the individual for whom risk is being calculated, the affected family members and the aggregation pattern in the family. It is important to recognize that a process of inference is used to produce individualized risks and that making a diagnosis of risk based on family history and other factors is not equivalent to making a diagnosis of disease based on the presence of certain symptoms. The distinction should be reflected in the language used by genetic counsellors. The primary reason why this distinction is so important is to resist the growing trend of identifying unaffected individuals as "patients without symptoms".

The paper by Austin and Peay ${ }^{14}$ provides a full and detailed discussion of the limitations of empiric risks, and of the theory behind individualization of these risks.

The characteristics of the individual for whom risk is being calculated that may be pertinent to individualized risk assessment include age, gender and psychiatric history. Characteristics of the affected family members that may be pertinent to risk assessment include gender, age at illness onset, and severity of illness. Factors relating to the aggregation pattern in the family that may be pertinent include bilineality, number and proportion of affected family members, and types of psychiatric diagnosis.

\section{Individualizing risks}

Unfortunately, empiric risks from available studies may not reflect the risks in individual families; those risks may be far higher or lower depending on the mode or transmission in a given family and the particular configuration and comorbidities of affected family members. Several factors may help determine whether particular families may be at the higher or lower range of risk:

- the presence of a greater number of affected family members is often thought to represent a more highly genetic form of the disorder and thus a higher risk of disease recurrence

- early onset of a disorder may reflect greater genetic loading

- gender of affected and at-risk family members
Given the variability and complexity of factors that may influence risk for a given family, it may not be possible to provide individualized risks in most instances. It is important to highlight the limitations of the information and to discuss how risks are derived, the spectrum of the diagnoses assessed in the supporting family studies, and the differences between empiric and individualized risks. It is most helpful to provide a range of risk, accompanied by the above qualifications. It is also important to remember that the perceived magnitude and impact of risks and probabilities may vary greatly from person to person. ${ }^{14}$

After genetic counselling there should be an improved understanding of the following issues:

- the complex nature of the genetic and environmental basis of psychiatric disorders

- the importance of accurate diagnoses for recurrence risk counselling

- an understanding of the limitations of empiric risks

- specific information about psychiatric disorder of interest (recurrence risks, symptoms, course of illness, treatment options)

- opportunities for decision making based on risk information

- sources of additional information and support related to being at risk or passing on risks to other family members

A comprehensive discussion of genetic risks for psychiatric disorders may be time-consuming and beyond the scope of practice for many psychiatrists. For patients who have questions beyond the knowledge base of their psychiatrists referral for consultation by genetics professional is appropriate. ${ }^{14}$

\section{The prospects of genetic testing}

Will genetic testing be feasible for psychiatric illness? In theory there is a scenario in which testing could be applied in schizophrenia. It involves velocardiofacial syndrome (VCFS) caused by a microdeletion of chromosome 22ql l that occurs in 1/4000 live births. In addition to a variety of physical features individuals with VCFS have elevated risks of psychiatric symptoms. In particular, rates of psychotic disorders have been reported to be as high as $25-30 \%$.

\section{Deletion of 22q1 1 and schizophrenia}

Hemizygous deletion in the chromosome $22 \mathrm{q} l 1$ region is associated with VCFS. These 1.5 - 3-Mb deletions are usually de novo events. Approximately 70\% of 22 ql 1 deletions are de novo; of the remaining 30\%, more than three quarters are maternally inherited. ${ }^{15}$

Hemizygosity of the $22 \mathrm{q} 11.2$ region seems to confer the major CNV risk for the expression of schizophrenia in 22q1 1.2DS. While further investigations are required to determine molecular risk factors that mediate de novo $22 q 11.2$ events during gameto genesis, the research results to date reinforce the need for genetic counselling of individuals with 22q l l.2DS regarding the risk for schizophrenia. Further efforts are required to identify the $1 \%$ of patients with schizophrenia who carry 22 ql 1.2 deletions. ${ }^{16}$ 
Deletion in the chromosome 22 ql 1 region is also a risk factor for schizophrenia. Patients with schizophrenia have been observed to have increased rates of 22 ql 1 deletion. Estimates range from $0.2 \%$ to $5.3 \%$. The estimated rate in Afrikaner schizophrenia patients was $2 \%$. In the general population this rate is $0.025 \%$. Estimates of psychosis in adult VCFS patients with 22 ql l deletion range from 6\% to 16\%. A recent study estimated the rate of DSM-IV schizophrenia diagnosis in 78 adults with 22 ql l deletion syndrome to be $22.6 \%$. This is a more than 20 -fold increase over the rate of schizophrenia in the general population. The mechanism by which this deletion contributes to the risk for schizophrenia and psychosis in VCFS patients is not yet understood. Proline dehydrogenase, a gene in the 22 ql 1 deletion interval, was reported to be associated with schizophrenia. Other reports suggested this gene is not associated with schizophrenia. The deletions identified in this region $-3 \mathrm{Mb}$ and $1.5 \mathrm{Mb}$ - are large and can be identified by standard cytology techniques (e.g. fluorescence in situ hybridibation - FISH). Until recently, technology to detect smaller deletions was unavailable. With newly available technology and decreasing resolution of detectable CNV size, it is possible that other structural variants in this region, such as smaller deletions or even duplications, will be identified. If additional variants are identified, they may serve to ultimately define a critical region for schizophrenia susceptibility with the 22ql l locus. This region is the most likely candidate region to harbor CNVs that contribute to schizophrenia susceptibility.15

Genetic counsellors can help to recognize when psychiatric disorders within a particular family may in fact be syndromic and due to an identifiable cause such as 22 ql l deletion.

In a study to assess the frequency of $22 \mathrm{q} 11$ deletion in Afrikaner schizophrenic patients the authors provided a twostage screening protocol to identify schizophrenia patients with this deletion. ${ }^{17}$ The screening of this sample for clinical features compatible with the 22 q I IDS was relatively simple to perform, accurately identified patients with the 22ql IDS and therefore considerably narrowed the sample size in need of confirmation by FISH. One third of the "flagged" cases were true positives and there were no false negatives. The criteria used, as suggested by Bassett and Chow are useful despite differences in their specificity and predictive value regarding $22 q 1$ lDS. ${ }^{18}$

According to Bassett and Chow (1999) ${ }^{18}$ patients with 2 or more of the following groups of screening criteria are at increased risk for 22ql lDS:

1. Hypernasal speech, history of speech therapy, velopharyngeal incompetence, cleft palate (usually submucosal)

2. Characteristic facial features e.g. long narrow face, narrow palpebral fissures, flat cheeks, prominent nose, small ears, small mouth, retruded chin

3. Learning difficulties, history of special education, MR (borderline to mild)

4. Congenital heart defects e.g. ventricle septum defect, tetralogy of Fallot, right Aortic arch, double aortic arch

5. Other significant congenital anomalies e.g. club feet, polydactily, renal anomalies, kyphosis/scoliosis, hypospadia
6. History of hypocalcaemia, and/or hypoparathyroidism

7. History of athymia or severe immunodeficiency in infancy

Of the 85 subjects with schizophrenia examined, 27 had one or more of the physical anomalies associated with the 22 ql lDS. ${ }^{17}$ Of these, 6 patients (four males, 2 females) had two or more of the positive screening criteria. A FISH analysis was performed on these six patients and two (one male, one female) were found to carry hemizygous deletions on chromosome 22q1 1. In an independent set of studies the entire sample was genotyped using a panel of single nucleotide polymorphismo (SNPs) from the 22 ql 1 locus. In the group of 85 schizophrenic patients described in this study, the same two individuals identified by FISH as carrying the 22ql 1 deletion were apparently "homozygous" for all tested markers. The rest of the patients were heterozygous for at least one marker locus, thus excluding the presence of a typical 22ql 1 microdeletion. Therefore it was concluded that the rate of 2 in 85 represents an accurate estimate and supports the conclusion that the accuracy of the clinical screening for 22ql lDS, as performed in this study, is 33\%. ${ }^{17}$

Physical and intellectual phenotypic features will remain the primary means of identifying a 22ql lDS subtype of schizophrenia. It is apparent that many individuals with 22qDS-schizophrenia will not have obvious congenital anomalies and that the majority of such individuals will not have mental retardation, although learning difficulties may be prevalent. This is consistent with the fact that individuals with 22qDS schizophrenia have been recruited into research samples despite intensive pre-screening, as illustrated by the National Institute of Mental health study of childhood-onset schizophrenia. Lower rates of comorbid substance use disorders may further facilitate the potential inclusion of subjects with 22qDS-schizophrenia in research studies of schizophrenia. A high index of suspicion, careful medical history taking and assessment for the more subtle physical and cognitive features of the syndrome will often be necessary to identify subjects with $22 \mathrm{qDS}$. Separate studies of these individuals will be important to determine which features distinguish this etiologic subtype from other forms of schizophrenia. ${ }^{19}$

\section{Genetic discrimination in mental disorders}

As discussed, genetic tests related to psychiatric disorders and their treatments are becoming more prominent in research and in the near future will be more so in clinical settings too. The possibility becomes more troubling that such information will be used for purposes other than those for which it was collected. For these reasons, the stages implementation of the federal Genetic Information nondiscrimination Act (GINA) of 2008 in the USA is of substantial importance to persons with mental disorders, persons at risk for the conditions, and family members of both group. ${ }^{20}$ The final provisions of the bill are due to have taken effect in 2010. GINA's major sections, Titles I and II, address health insurance and employment contexts, respectively. Title I bars health insurers from requesting genetic information from clients, including requiring clients to undergo genetic tests, and from using genetic information obtained from other sources to make coverage decisions or to set premiums. In comparable fashion, Title II prohibits employers from 
mandating that workers be tested for genetic propensities to illness and from using genetic information that may otherwise come to their attention for a range of employment-related decisions and termination. Violations of either are subject to substantial monetary penalties. ${ }^{21}$

This type of legislation impacts on psychiatry and might be more limited than in other areas of medicine for several reasons. But as has been discussed earlier, family history information on many mental disorders can be quite useful in identifying risk categories for close relatives. In the USA when questions about genetic testing arise, clinicians should make every effort to ensure that their patients are aware of GINA and related state laws and that they have an accurate understanding of the protections that these regulations provide. In the SA context the Mental Health Care Act states in article 10 that "users" may not be unfairly discriminated against on grounds of mental illness or that users must receive the same health services as any health user. ${ }^{22}$ Further development of GINA related legislation in the SA context will be necessary in the future.

\section{Conclusion}

The recent advances in the field of genetic research and their wide publicity in the scientific and clinical journals as well as on the internet have created an ever rising demand for genetic counselling regarding schizophrenia. Quite often it is the psychiatrist who must convey this information as genetic counsellors feel uncertain when it comes to accurately making psychiatric diagnosis as well as the course and prognosis of illness. Furthermore, genetic counselling for complex disorders is among the most challenging areas encountered by genetics professionals and the role that environmental factors play are not clear cut either. The newest developments in understanding hereditary and other factors believed to contribute to the etiology, clinical presentation, course and prognosis of the disease should be taken into account when genetic counselling is requested. Psychiatrists and allied mental health and other professionals need to be vigilant in preventing genetic discrimination in mental disorders.

\section{References}

1. Finn CT, Smoller JW. Genetic Counseling in Psychiatry. Harv Rev Psychiatry 2006; 14:109-121.

2. Austin JC, Honer WG. The potential impact of genetic counselling for mental illness. Clin Genet 2005; 67:134-42.

3. Smith LB, Sapers B, Reus VI, Freimer NB. Attitudes towards bipolar disorder and predictive genetic testing among patients and providers. J Med Genet 1996; 33:544-9.
4. Smoller JW, Finn CT. Family, twin, and adoption studies of bipolar disorder. Am J Med Genet C Semm Med Genet. 2003; 123:48-58.

5. Tsuang MT, Stone WS, Faraone SV. Genes, environment and schizophrenia. Br J Psychiatry 2001; 178:518-24.

6. Tsuang M. Schizophrenia: genes and environment. Biol Psychiatry 2000; 47:210-20.

7. Harper PS. Practical genetic counselling. Oxford, Boston: Butterworth-Heinemann; 1998.

8. McGuffin P, Owen MJ, Gottesman II. Psychiatric genetics and genomics. Oxford, New York: Oxford University Press, 2002.

9. "Copy number variation: The new genetic frontier (Report)". Harvard Mental Health letter (Oct 1, 2008): NA Academic OneFile.

10. St Clair D. Copy Number Variation and Schizophrenia. Schizophrenia Bulletin 2009, 35(1):9-12.

11. Xu B, Woodroffe A, Rodriquez-Murillo L, Roos JL, J v Rensburg L, Abecasis GR, Gogos JA, Karayiorgou M. Elucidating the genetic architecture of familial schizophrenia using rare copy number variant and linkage scans. PNAS 2009:106(39):16746-16751.

12. Xu B, Roos JL, Van Rensburg EJ, Gogos JA, Karayiorgou M. Strong association of de novo copy number mutations with sporadic schizophrenia. Nat Genet 2008; 40 (7): 880-885.

13. Austin JC, Palmer CGS, Rosen-Sheidly B, Veach PM, Gettig E, Peay HL. Psychiatric Disorders in Clinical Genetics II: individualizeing Recurrence Risks. J Genet Cousel 2008; 17:18-29.

14. Austin JC, Peay HL. Applications and limitations of empiric data in provision of recurrence risks for schizophrenia: A practical review for healthcare professionals providing clinical psychiatric genetics consultations. Clinical Genetics 2006; 70(3):177-187.

15. Mulle J. Genomic Structural Variation and Schizophrenia. Current Psychiatry Reports 2008;10:171-177.

16. Bassett AS, Marshall CR, Lionel AC, Chow EWC, Scherer SE. Copy number variations and risk for schizophrenia in 22ql 1 deletion syndrome. Human Molecular Genetics 2008; 17(24):4045-4053.

17. Wiehahn GJ, Bosch GP, Du Preez RR, Pretorius HW, Karayiorgou M, Roos JL. Assessment of the Frequency of the 22ql 1 deletion in Afrikaner schizophrenia patients. American Journal of Medical Genetics Part B (Neuropsychiatric Genetics) 2004; 129B: 20-22.

18. Bassett AS, Chow EWC. 22q1 1 deletion syndrome: A genetic subtype of schizophrenia. Biol Psychiatry 1999; 46:882-891.

19. Bassett AS, Chow EWC, AbdelMalik P, Gheorghiu M, Husted J, Weksberg R. The Schizophrenia Phenotype in 22q1 1 Deletion Syndrome. Am J Psychiatry 2003; 160:1580-1586.

20. Genetic Information Nondiscrimination Act of 2008, Public Law 110 233, May 21, 2008.

21. Appelbaum PS. Genetic Discrimination in Mental Disorders: The impact of the Genetic Information non-discrimination Act. 2010. Psychiatric Services 2010; 61:338-340.

22. New Mental Health Care Act (Act 17 of 2002) Government Gazette No 27117:1-76. 